\title{
Identification of the Otopetrin Domain, a conserved domain in vertebrate otopetrins and invertebrate otopetrin-like family members
}

\author{
Inna Hughes ${ }^{\dagger 1}$, Jonathan Binkley ${ }^{\dagger 2}$, Belen Hurle ${ }^{\dagger 3}$, Eric D Green ${ }^{3,4}$, NISC \\ Comparative Sequencing Program ${ }^{3,4}$, Arend Sidow ${ }^{2}$ and David M Ornitz*1
}

Address: ${ }^{1}$ Department of Developmental Biology, Washington University School of Medicine, St. Louis, MO 63110, USA, ${ }^{2}$ Departments of Genetics and Pathology, Stanford University Medical Center, Stanford, CA 94305, USA, ${ }^{3}$ Genome Technology Branch, National Human Genome Research Institute, National Institutes of Health, Bethesda, MD 20892, USA and ${ }^{4}$ NIH Intramural Sequencing Center (NISC), National Human Genome Research Institute, National Institutes of Health, Bethesda, MD 20892, USA

Email: Inna Hughes - Inna_Hughes@urmc.rochester.edu; Jonathan Binkley - binkley@genome.stanford.edu; Belen Hurle - bhurle@nhgri.nih.gov; Eric D Green - egreen@nhgri.nih.gov; NISC Comparative Sequencing Program - egreen@nhgri.nih.gov; Arend Sidow - arend@stanford.edu; David M Ornitz* - dornitz@wustl.edu

* Corresponding author †Equal contributors

\section{Published: 6 February 2008}

BMC Evolutionary Biology 2008, 8:4| doi:|0.1 I86/I47|-2|48-8-4|
Received: 14 September 2007

Accepted: 6 February 2008

This article is available from: http://www.biomedcentral.com/|47|-2/48/8/4 I

(C) 2008 Hughes et al; licensee BioMed Central Ltd.

This is an Open Access article distributed under the terms of the Creative Commons Attribution License (http://creativecommons.org/licenses/by/2.0), which permits unrestricted use, distribution, and reproduction in any medium, provided the original work is properly cited.

\begin{abstract}
Background: Otopetrin I (Otop I) encodes a multi-transmembrane domain protein with no homology to known transporters, channels, exchangers, or receptors. Otopl is necessary for the formation of otoconia and otoliths, calcium carbonate biominerals within the inner ear of mammals and teleost fish that are required for the detection of linear acceleration and gravity. Vertebrate Otop 1 and its paralogues Otop2 and Otop3 define a new gene family with homology to the invertebrate Domain of Unknown Function 270 genes (DUF270; pfam03189).

Results: Multi-species comparison of the predicted primary sequences and predicted secondary structures of 62 vertebrate otopetrin, and arthropod and nematode DUF270 proteins, has established that the genes encoding these proteins constitute a single family that we renamed the Otopetrin Domain Protein (ODP) gene family. Signature features of ODP proteins are three "Otopetrin Domains" that are highly conserved between vertebrates, arthropods and nematodes, and a highly constrained predicted loop structure.

Conclusion: Our studies suggest a refined topologic model for ODP insertion into the lipid bilayer of 12 transmembrane domains, and highlight conserved amino-acid residues that will aid in the biochemical examination of ODP family function. The high degree of sequence and structural similarity of the ODP proteins may suggest a conserved role in the intracellular trafficking of calcium and the formation of biominerals.
\end{abstract}

\section{Background}

Otopetrin1 (Otop1) is the first described member of the otopetrin family, a novel gene family that encodes multi- transmembrane domain proteins. The family was named for the conserved role of Otop1 in the formation of otoconia and otoliths - "oto" (ear) and "petros" (stone). Oto- 
conia are complex calcium carbonate biominerals in the utricle and saccule of the vertebrate inner ear that are required for the normal sensation of linear acceleration and gravity. Degeneration or displacement of otoconia can lead to vertigo and loss of balance [1-5]. Three mutant mice and one zebrafish model with mutations in Otop1 have been described: tilted (tlt) [6]; mergulhador ( $\mathrm{mlh}$ ) [7]; inner ear defect (ied) [8]; and backstroke (bks) [9], respectively. All of these mutants lack otoconia or otoliths, but have normal inner ear development. In zebrafish, the morpholino knockdown of Otop1 phenocopies the tlt mutation, showing otolith agenesis with no disruption of the patterning of the developing inner ear $[9,10]$.

The otopetrin family in most vertebrates studied consists of three genes clustered in two chromosomal locations: Otop1 (i.e., human Chr 4p16, mouse Ch5B2) and the paralogous tandem genes Otop2 and Otop3 (i.e., human Ch17q24-25, mouse Ch11E2). Vertebrate otopetrins share a conserved gene and protein structure, with no homology to other transporters, channels, exchangers, or receptors. A preliminary secondary structure prediction based on the human, mouse, rat, zebrafish, and fugu protein sequences suggested a topology of ten transmembrane domains (TM) with cytosolic amino and carboxy termini. Additionally, tBlastn searches in the EST and genomic databases identified regions of homology with the DUF270 domain in a number of arthropod and nematode proteins. DUF270 (pfam03189) is a 404 aminoacid consensus sequence of unknown function that defines the DUF270 family, with members in C. elegans and $D$. melanogaster. The two regions of maximum homology with DUF270 found in vertebrate otopetrins correspond to putative TM domains 3-5 and 9-10, respectively, and were initially designated DUF270-I and DUF270-II [7].

Here, we report a comparison of evolutionary constraint and hydropathy profile analysis of 62 vertebrate otopetrins and arthropod and nematode DUF270 proteins, demonstrating that the genes that encode these proteins constitute a single family that we have renamed the Otopetrin Domain Protein $(O D P)$ gene family. The refined topologic model of the ODP proteins includes 12 putative TM domains clustered into three "Otopetrin Domains" (OD-I, -II, and -III, respectively), with a strong degree of sequence conservation across widely divergent groups of metazoa. These regions of highest homology and evolutionary constraint, including the FYR box in the cytoplasmic tail, may represent important functional subdomains. Biochemical studies in transfected cells show that Otop1 modulates the manner in which cells handle intracellular calcium in response to purinergic stimuli [11]. The lack of known functional domains, such as ATPbinding domains, selectivity pores, or G-protein-binding consensus sequences, suggests that either the ODP family has a novel function that significantly differs from the activities of known channels, transporters, or receptors, or that the ODP genes encode novel functional motifs. We hypothesize that these motifs would likely occur within the evolutionarily constrained regions, as has been shown for other well-conserved gene families [12]. The challenge remains to define the functional domains of the ODP family, with sequence and analyses reported here providing a step in that direction.

\section{Results and Discussion}

\section{Comparative sequence data set}

The annotation of the Otop1, Otop2, and Otop3 genes in the human, mouse, rat, zebrafish, and fugu genomes is described elsewhere [7]. Orthologous otopetrin sequences were generated using a targeted sequencing approach (from dog, cow, armadillo and western clawed frog) (see methods in $[13,14]$ ) or identified through tBlastn searches of available whole-genome sequences. The phylogenetic relationships of vertebrate otopetrin and arthropod and nematode DUF270 genes were deduced from a total of 62 complete or nearly complete open reading frames in 25 species (see Table 1 for a listing of the specific species and accession numbers). Fragmentary, but clearly otopetrin-related, sequences were also identified in urochordates (ciona), echinoderms (urchin), and cnidarians (nematostella), however were not complete enough to include in this analysis.

\section{Phylogenetic relationships and revised nomenclature of vertebrate otopetrins and arthropod and nematode DUF270 genes}

A maximum-likelihood phylogenetic tree was created from the multi-sequence alignment of each encoded protein (Figure 1). The vertebrate, arthropod, and nematode sequences form distinct monophyletic groups, each containing three or more paralogous groups. This arrangement suggests that the ancestral metazoan genome may have contained a single otopetrin-like gene, with subsequent duplications giving rise to the paralogs in the different phyla after the three lineages diverged. Based on the positions in the tree of the named mouse and human sequences, the three vertebrate paralogous groups correspond to Otop1, Otop2, and Otop3. Otop2 and Otop3 are more closely related to each other than either is to Otop1, a clustering that parallels the genomic organization of the Otop genes in the vertebrate genomes. The arthropod and nematode DUF270 sequences, in which encoded proteins cluster independently in the tree from the vertebrate otopetrin sequences, have been renamed as otopetrin-like proteins (OTOPL), and the paralogous groups have been assigned arbitrary letters. This is in agreement with the HUGO gene nomenclature committee guidelines for gene families and grouping [15]. Like verte- 
Table I: Otopetrin Domain Protein genes

\begin{tabular}{|c|c|c|c|c|}
\hline Species & Name & Gene & Symbol & Accession No. \\
\hline \multirow[t]{3}{*}{ Human } & Homo sapiens & otopetrin I & OTOPI & NM_I77998 \\
\hline & & otopetrin 2 & OTOP2 & NM_I78I60 \\
\hline & & otopetrin 3 & OTOP3 & NM_I78233 \\
\hline \multirow[t]{2}{*}{ Chimpanzee } & Pan troglodytes & otopetrin I & Otop I & * ENSPTRT00000029625 \\
\hline & & otopetrin 2 & Otop2 & XM_5II667 \\
\hline Rhesus macaque & Macaca mulatta & otopetrin I & Otopl & XM_001097009 \\
\hline \multirow{3}{*}{ Mouse } & Mus musculus & otopetrin I & Otopl & NM_172709 \\
\hline & & otopetrin 2 & Otop2 & NM_I7280I \\
\hline & & otopetrin 3 & Otop3 & NM_027I32 \\
\hline \multirow[t]{3}{*}{ Rat } & Rattus norvegicus & otopetrin I & Otopl & NM_I81433 \\
\hline & & otopetrin 2 & Otop2 & XM_221107 \\
\hline & & otopetrin 3 & Otop3 & XM_00108I677 \\
\hline Cow & Bos taurus & otopetrin 2 & Otop2 & XM_606240, ACI48430 \\
\hline \multirow[t]{2}{*}{ Dog } & Canis familiaris & otopetrin 2 & Otop2 & XM_540422, ACI49469 \\
\hline & & otopetrin 3 & Otop3 & XM_540423, ACI49469 \\
\hline \multirow[t]{2}{*}{ Opossum } & Monodelphis domestica & otopetrin 2 & Otop2 & * ENSMODT00000008924 \\
\hline & & otopetrin 3 & Otop3 & * ENSMODG00000007075 \\
\hline Platypus & Ornithorhynchus anatinus & otopetrin 3 & Otop3 & * ENSOANG00000004377 \\
\hline Armadillo & Dasypus novemcinctus & otopetrin 2 & Otop2 & $\mathrm{ACI} 47459$ \\
\hline \multirow[t]{3}{*}{ Western clawed frog } & Xenopus tropicalis & otopetrin I & Otop I & * ENSXETT00000055844 \\
\hline & & otopetrin 2 & Otop2 & * ENSXETP000000I4996 \\
\hline & & otopetrin 3 & Otop3 & $\mathrm{ACl} 166187$ \\
\hline \multirow[t]{2}{*}{ Chicken } & Gallus gallus & otopetrin I & Otop I & * ENSGALP00000024I 28 \\
\hline & & otopetrin 3 & Otop3 & XM_420II5 \\
\hline Japanese medaka & Oryzias latipes & otopetrin I & Otopl & * ENSORLT000000104I4 \\
\hline Zebrafish & Danio rerio & otopetrin I & Otop I & NM_198803 \\
\hline Tetraodon & Tetraodon nigroviridis & otopetrin I & Otop I & † CAAEOI0I4674 (CAG02008) \\
\hline Three-spined & Gasterosteus aculeatus & otopetrin I & Otopl & * ENSGACT000000I2102 \\
\hline \multirow[t]{2}{*}{ stickleback } & & otopetrin 2 & Otop2 & * ENSGACT000000I4538 \\
\hline & & otopetrin 3 & Otop3 & * ENSGACT00000019137 \\
\hline \multirow[t]{2}{*}{ Fugu } & Fugu rubripes & otopetrin I & Otopl & BK000652 \\
\hline & & otopetrin 3 & Otop3 & * SINFRUT00000 I 403 II \\
\hline Yellow fever & Aedes aegypti & otopetrin-like bl & OTOPLbI & † CH4773I2 (EAT43886) \\
\hline \multirow[t]{2}{*}{ mosquito } & & otopetrin-like b2 & OTOPLb2 & † CH4773I 2 (EAT43887) \\
\hline & & otopetrin-like c & OTOPLC & † CH477407 (EAT4I549) \\
\hline \multirow[t]{3}{*}{ Fruitfly } & Drosophila melanogaster & otopetrin-like a & OTOPLa & AY07I5I0 \\
\hline & & otopetrin-like b & OTOPLb & NM_I6453I \\
\hline & & otopetrin-like c & OTOPLC & NM_I32010 \\
\hline \multirow[t]{2}{*}{ Fruitfly } & Drosophila & otopetrin-like b & OTOPLb & † CH37906I (EAL32988) \\
\hline & pseudoobscura & otopetrin-like c & OTOPLC & † CH379063 (EAL32758) \\
\hline \multirow[t]{2}{*}{ Honey bee } & Apis mellifera & otopetrin-like a & OTOPLa & XM_394295 \\
\hline & & otopetrin-like c & OTOPLC & XM_394296 \\
\hline \multirow[t]{4}{*}{ Malaria mosquito } & Anopheles gambiae & otopetrin-like a & OTOPLa & XM_31I233 \\
\hline & & otopetrin-like bl & OTOPLbI & XM_311078 \\
\hline & & otopetrin-like b2 & OTOPb2 & XM_311079 \\
\hline & & otopetrin-like c & OTOPLC & XM_31I232 \\
\hline Red flour beetle & Tribolium castaneum & otopetrin-like a & OTOPLa & XM_969602 \\
\hline & & otopetrin-like b & OTOPLb & XM_96280I \\
\hline & & otopetrin-like c & OTOPLC & XM_969568 \\
\hline Nematode & Caenorhabditis & otopetrin-like $d$ & OTOPLd & † CAAC0I000008 (CAE58380) \\
\hline & briggsae & otopetrin-like e & OTOPLe & † CAAC0I000008 (CAE5838I) \\
\hline & & otopetrin-like f & OTOPLf & † CAAC0I000008 (CAE58382) \\
\hline & & otopetrin-like g & OTOPLg & † CAAC0I000076 (CAE69908) \\
\hline & & otopetrin-like h & OTOPLh & † CAAC0I000035 (CAE63792) \\
\hline & & otopetrin-like i & OTOPLi & † CAAC0I000052 (CAE658I9) \\
\hline Nematode & Caenorhabditis & otopetrin-like dl & OTOPLdI & t† U64845 (AAC48028) \\
\hline & elegans & otopetrin-like $\mathrm{d} 2$ & OTOPLd2 & NM_07I735 \\
\hline & & otopetrin-like e & OTOPLe & t† U64845 (AAC48027) \\
\hline & & otopetrin-like f & OTOPLf & t† U64845 (AAC48029) \\
\hline & & otopetrin-like g & OTOPLg & t†t AL009I 70 (CAAI 5637) \\
\hline & & otopetrin-like h & OTOPLh & t† AF045639 (AAC02566) \\
\hline & & otopetrin-like i & OTOPLi & †† U28737 (AAL02486) \\
\hline
\end{tabular}

In some instances, the nucleotide accession number corresponds to a tscaffold, ttcosmid, or tttfosmid record; in those cases, the accession number of the Otop or OTOPL annotation (protein) is indicated in parenthesis.

* ENSEMBL accession number 
brates, arthropods also have three paralogous groups of OTOPLs. The grouping in nematodes is more complex: there appears to be three major groups of OTOPLs, as in vertebrates and arthropods, but each group itself contains two or more paralogous groups as a result of species-specific gene duplications. In summary, vertebrate otopetrins and arthropod and nematode OTOPL genes have been grouped as a single family that we named collectively the Otopetrin Domain Protein (ODP, see below) gene family.

\section{Refined topological model for ODP insertion into the lipid bi-layer}

Conserved primary sequence is indicative of an underlying conserved tertiary structure, and the evolutionary information contained in an alignment of related sequences can be leveraged to improve predictions of shared structures [16]. We took advantage of the deep multi-sequence alignment and phylogenetic tree of the ODP family to reexamine the predicted topology of the ODPs (Figure 2A). A hydropathy profile was generated that employs phylogenetic averaging [17] on hydropathy scale values for amino acids [18] to improve the detection of conserved hydrophobic regions, which might correspond to TM domains. The hydropathy profile revealed 12 strong hydrophobic regions, ten of which overlap with the originally predicted TM domains [7]. Likewise, the MEMSAT3 [19] and TMAP [20] algorithms, which take into account leveraged evolutionary information, also predicted 12 TM helices for ODP family members that overlap well with the constrained regions and hydrophobic regions in our profile (Figure 2A).

The refined topological model for the ODP family thus consists of 12 TM domains, with both the $\mathrm{N}$ - and C-termini in the cytosol, and in which the two newly identified TM domains are TM4 and TM10, respectively. As shown in Figure $2 \mathrm{~B}$, there are three discrete regions with maximum evolutionary constraint among vertebrates, arthropods and nematodes, which we have designated Otopetrin Domain (OD) -I, -II, and -III, respectively. Among the TM domains, TM2 and TM8 show the poorest conservation and evolutionary constraint across species. On the other hand, the loops connecting the TM domains show little sequence conservation or evolutionary constraint, strongly suggesting that the TM domains are the primary functional regions of the ODP family (Figure 2A and Additional file 1). Despite the poor loop sequence conservation, the number of amino acids in 8 of the 11 loops separating TM domains is highly conserved (Table 2), suggesting that the spacing of most of the TM domains relative to one another may be important for the tertiary structure and function of ODP family members. Of note, the length of loop 5, within OD-I, is highly variable across all phyla, but conserved in vertebrates $(48 \pm 4$ amino acid residues), as are all other loops except for loop 10.

\section{Homology between Otop and OTOPL sequences extends beyond the canonical DUF270 domain}

DUF270 (pfam03189) is a 404 amino-acid consensus sequence of unknown function. Early tBlastn-based database searches identified regions of homology with the DUF270 domain in both vertebrate Otop and arthropod and nematode OTOPL proteins [7], now grouped together as the ODP family. Inspection of the multi-species ODP sequence alignment suggests that the homology among ODP proteins extends beyond the canonical DUF270 domain (see Additional file 1). Specifically, the $\mathrm{N}$-terminal end of the DUF270 consensus sequence can be extended to include three amino acids (HAG, amino acids 125-127 in mouse Otop1) that are conserved in most vertebrate (HAG) and nematode (GAG) ODPs examined. At the C-terminal end, the amino-acid conservation continues well beyond the DUF270 motif to include the entire C-terminal tail of vertebrate Otop (amino acids 584-600 in mouse Otop1). A 14-aminoacid consensus sequence for this highly conserved C-terminal tail, which we named the FYR box, is shown in Figure 3 . The FYR box is a signature unique to the ODP family, and is present in all ODP proteins but not in any non-ODP sequences in the databases of ESTs and nonredundant sequences.

\section{Conclusion}

Comparative analyses of vertebrate otopetrins and arthropod and nematode OTOPL proteins revealed that they all share a TM domain structure and significant conservation of amino-acid sequence, suggesting that they constitute a single protein family, here renamed the ODP family. We have expanded the domains of homology to more accurately reflect the extent of sequence conservation between vertebrates, arthropods and nematodes, and have identified three evolutionarily constrained TM domain-rich areas that we have designated as Otopetrin Domains.

OD-I and OD-III are the most highly conserved regions of the ODP family. Tlt mice carry a missense mutation $\left(\mathrm{Ala}_{151} \rightarrow \mathrm{Glu}\right)$, which alters the hydrophobicity of the predicted TM3 domain within OD-I, and leads to a presumed alteration in the membrane insertion or activity of Otop1 and otoconial agenesis [7]. The OD-II evolutionarily constrained region was not identified in the initial modeling, but mutations in Otop1 within this conserved segment of the protein have been shown to cause otolith/otoconial agenesis in bks mutant fish ( $\left.\mathrm{Glu}_{429} \rightarrow \mathrm{Val}\right)$ [9] and in $\mathrm{mlh}$ mutant mice ( Leu $_{408} \rightarrow \mathrm{Gln}$ ) [7] (Figure 2B), suggesting that this region is functionally important.

Initial modeling of the OTOP proteins suggested a $10 \mathrm{TM}$ domain model with cytosolic N- and C-termini [7]. This model had several problems, including that sites consistent with the consensus sequence for $\mathrm{N}$-glycosylation were 


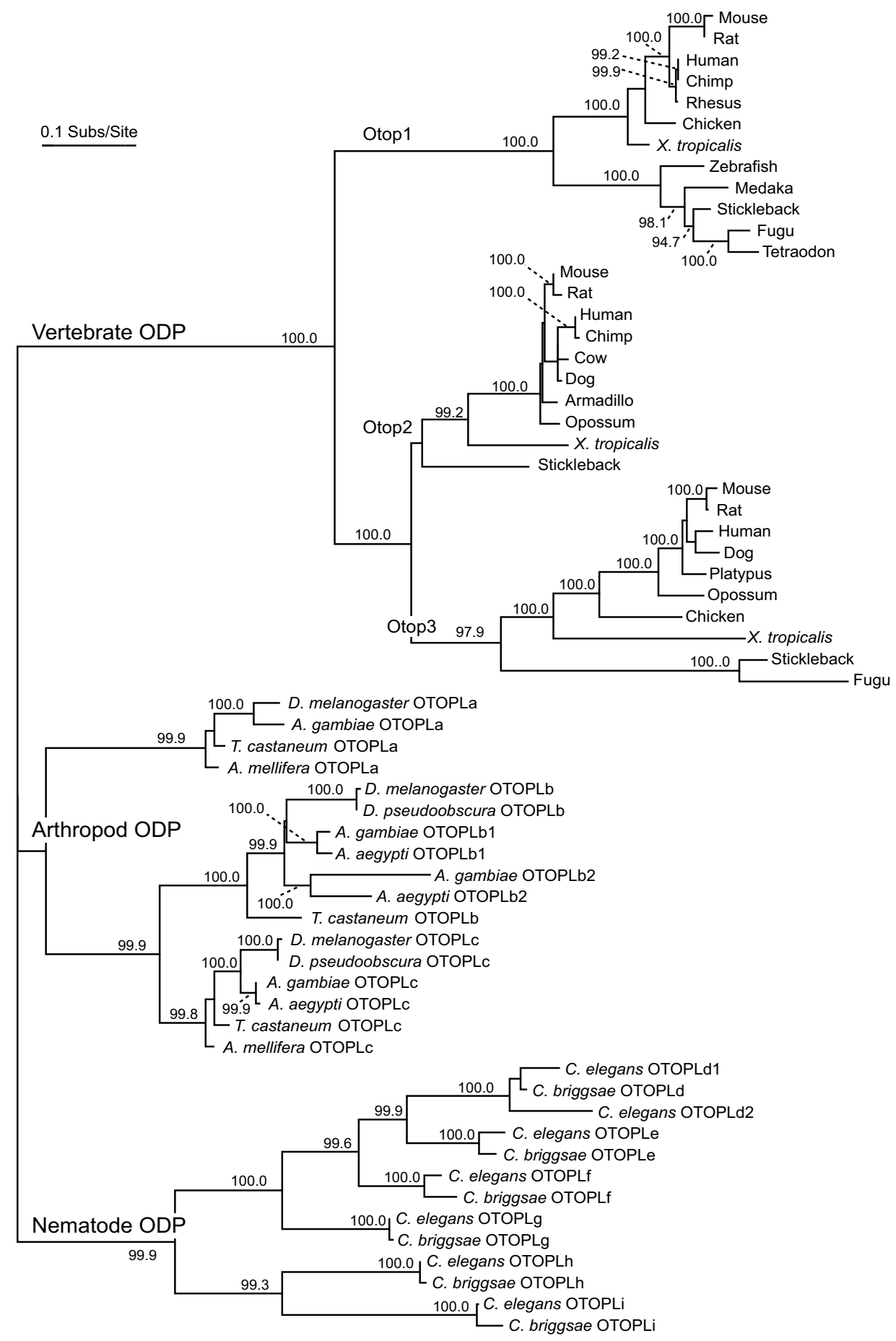

Figure I

Phylogeny of the Otopetrin Domain Protein (ODP) family. Maximum-likelihood phylogenetic tree created from the multi-sequence alignment of 62 ODPs (see additional file I). The vertebrate, arthropod, and nematode sequences form distinct monophyletic groups, each containing three or more paralogous groups. Some nematode and arthropod sequences appear to have undergone additional gene-duplication events, creating species-specific paralogs (designated with a I or 2 following the gene symbol). Branch labels are bootstrap values for 1000 replicates. Unlabeled internal branches have bootstrap values less than 90.0. 

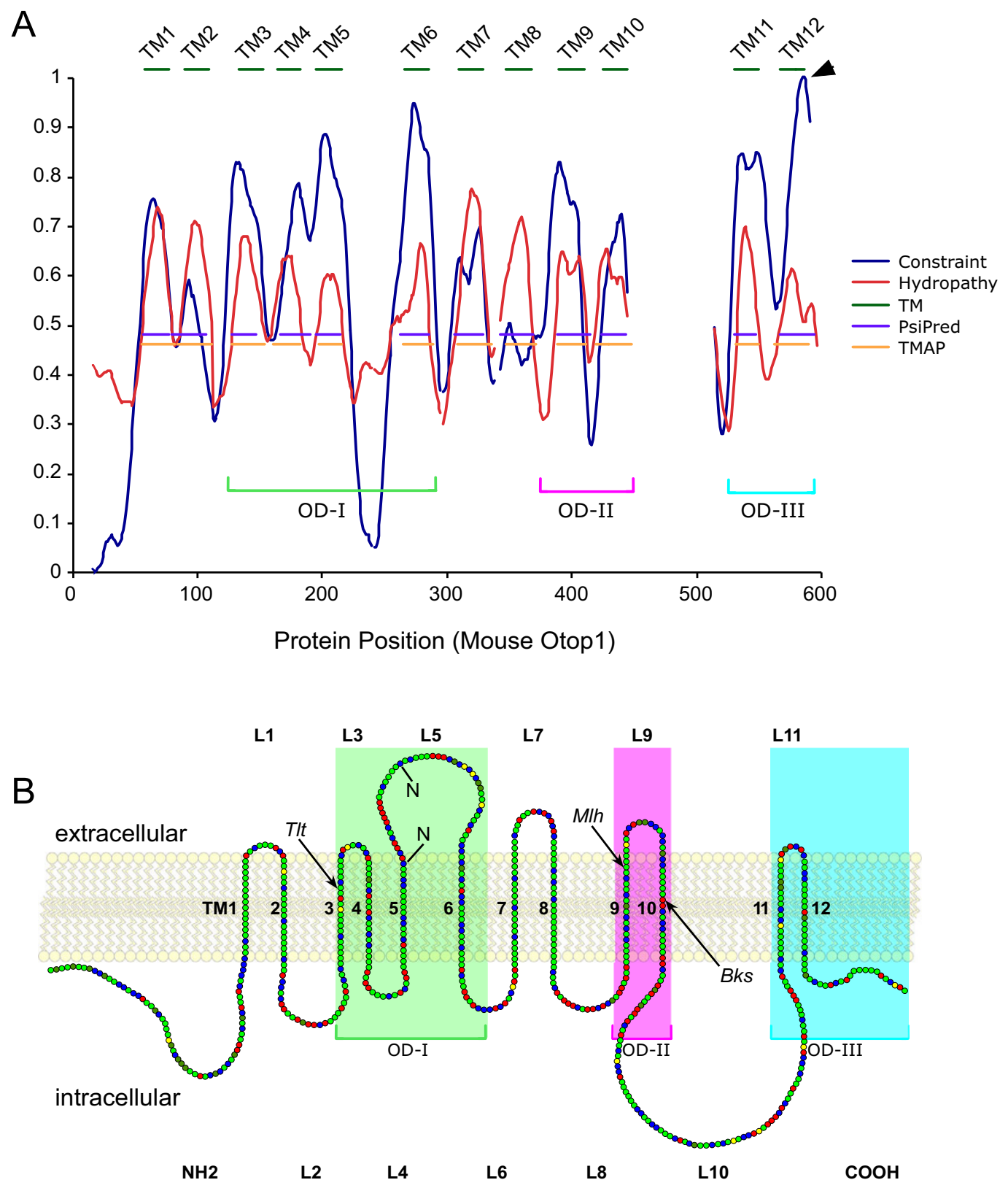

\section{Figure 2}

Predicted secondary structure and topologic model for Otop I insertion into the lipid bilayer. A) Hydrophobicity (red) and evolutionary constraint (blue) are plotted against the amino-acid position of mouse Otop I. A total of 12 evolutionarily constrained regions are found in the ODP family that are highly hydrophobic and have a helical structure consistent with TM domains (dark green), as predicted by TMAP (orange) and PsiPred (purple). Green, pink, and blue brackets define the highly conserved subdomains: Otopetrin Domain-I, -II, and -III (OD-I, OD-II, and OD-III, respectively). B) Linear model of mouse Otop la inserted in a lipid bilayer, in which each amino acid is represented as a circle and the chemical properties of aminoacids are denoted by color: charged residues (red), polar residues (blue), and non-polar residue (green). Cysteine (yellow) and proline (dark green) are noted. The two consensus $\mathrm{N}$-glycosylation sites $(\mathrm{N})$ are indicated in loop 5 . The predicted intracellular and extracellular loops and TM domains are numbered LI to LII and TMI to TMI2, respectively. The locations of the tit, mlh, and bks mutations are noted by arrows. The three OD subdomains are shaded with the color code used in $A$. 
Table 2: Transmembrane domain inter-loop length (amino-acids)

\begin{tabular}{|c|c|c|c|c|c|c|c|c|c|c|c|c|c|}
\hline & $\mathrm{NH}_{2}(\mathrm{I})$ & LI (O) & L2 (I) & L3 (0) & L4 (I) & L5 (0) & L6 (I) & L7 (0) & L8 (I) & L9 (0) & LIO (I) & LII (O) & COOH (I) \\
\hline Mouse Otopl & $58 \#$ & $12 \#$ & 23 & 10 & 11 & 50 & 21 & 9 & 19 & 11 & 85 & 16 & I3\# \\
\hline Rat Otop I & 58 & 12 & 23 & 10 & 11 & 50 & 21 & 9 & 19 & 11 & 85 & 16 & 13 \\
\hline Human OTOPI & 61 & 12 & 23 & 10 & 11 & 50 & 21 & 9 & 19 & 11 & 95 & 16 & 13 \\
\hline Chimp Otopl & 61 & 12 & 23 & 10 & 11 & 50 & 21 & 9 & 19 & 11 & 95 & 16 & 13 \\
\hline Rhesus Otop I & 61 & 12 & 23 & 10 & 11 & 50 & 21 & 9 & 19 & 11 & 93 & 16 & 13 \\
\hline Chicken Otopl & $11^{*}$ & 12 & 23 & 10 & 11 & 50 & 21 & 9 & 19 & 11 & 98 & 16 & 13 \\
\hline X. tropicalis Otop I & 39 & 12 & 23 & 10 & 11 & 47 & 21 & 9 & 19 & 11 & 96 & 16 & 13 \\
\hline Zebrafish OtopI & 54 & 12 & 23 & 10 & 11 & 47 & 22 & 9 & 19 & 11 & 75 & 16 & 13 \\
\hline Medaka OtopI & 52 & 12 & 23 & 10 & 11 & 49 & 22 & 9 & 19 & 11 & 73 & 16 & 13 \\
\hline Stickleback Otop I & 51 & 12 & 24 & 10 & 11 & 47 & 22 & 9 & 19 & 11 & 87 & 16 & 13 \\
\hline Fugu Otopl & 51 & 12 & 23 & 10 & 11 & 47 & 22 & 9 & 19 & 11 & 87 & 16 & 13 \\
\hline Tetraodon Otop I & 50 & 12 & 23 & 10 & 11 & 47 & 22 & 9 & 19 & 11 & 87 & 16 & 12 \\
\hline Mouse Otop2 & 30 & 12 & 23 & 10 & 11 & 54 & 26 & 9 & 19 & 11 & 67 & 16 & 13 \\
\hline Rat Otop2 & 30 & 12 & 23 & 10 & 11 & 54 & 26 & 9 & 19 & 11 & 67 & 16 & 13 \\
\hline Human OTOP2 & 30 & 12 & 23 & 10 & 11 & 53 & 26 & 9 & 19 & 11 & 67 & 16 & 13 \\
\hline Chimp Otop2 & 30 & 12 & 23 & 10 & 11 & 53 & 26 & 9 & 19 & 11 & 67 & 16 & 13 \\
\hline Dog Otop2 & 30 & 12 & 23 & 10 & 11 & 53 & 26 & 9 & 19 & 11 & 67 & 16 & 13 \\
\hline Cow Otop2 & 30 & 12 & 23 & 10 & 11 & 53 & 26 & 9 & 19 & 11 & 67 & 16 & 13 \\
\hline Armadillo Otop2 & 30 & 12 & 23 & 10 & 11 & 53 & 26 & 9 & 19 & 11 & 67 & 16 & 13 \\
\hline Opossum Otop2 & 30 & 12 & 23 & 10 & 11 & 46 & 32 & 6 & 19 & 11 & 65 & 16 & 13 \\
\hline X. tropicalis Otop2 & $31^{*}$ & 12 & 22 & 10 & 11 & 54 & 25 & 8 & 19 & 11 & 62 & 16 & 13 \\
\hline Stickleback Otop2 & $3 I^{*}$ & 12 & 23 & 10 & 11 & 52 & 20 & 12 & 19 & 11 & 64 & 16 & 13 \\
\hline Mouse Otop3 & 70 & 12 & 23 & 10 & 11 & 44 & 24 & 8 & 19 & 11 & 54 & 16 & 13 \\
\hline Rat Otop3 & 71 & 12 & 23 & 10 & 11 & 44 & 24 & 8 & 19 & 11 & 54 & 16 & 13 \\
\hline Human OTOP3 & 89 & 12 & 23 & 10 & 11 & 44 & 24 & 8 & 19 & 11 & 54 & 16 & 13 \\
\hline Dog Otop3 & 71 & 12 & 23 & 10 & 11 & 44 & 24 & 8 & 19 & 11 & 54 & 16 & 13 \\
\hline Opossum Otop3 & $59 *$ & 12 & 23 & 10 & 11 & 44 & 24 & 8 & 19 & 11 & 58 & 16 & 13 \\
\hline Platypus Otop3 & $5 I^{*}$ & 12 & 23 & 10 & 11 & 44 & 24 & 8 & 19 & 11 & 51 & 16 & 13 \\
\hline Chicken Otop3 & 24 & 12 & 23 & 10 & 11 & 44 & 23 & 8 & 19 & 11 & 62 & 16 & 13 \\
\hline X. tropicalis Otop3 & 34 & 12 & 23 & 10 & 11 & 43 & 22 & 8 & 19 & 11 & 46 & 16 & 13 \\
\hline Stickleback Otop3 & 48 & 12 & 23 & 10 & 11 & 45 & 28 & 8 & 19 & 11 & 60 & 16 & 13 \\
\hline Fugu Otop3 & $I^{*}$ & 12 & 23 & 10 & 11 & 45 & 27 & 8 & 19 & 11 & 44 & 16 & 11 \\
\hline $\begin{array}{l}\text { D. melanogaster } \\
\text { OTOPLa }\end{array}$ & 64 & 14 & 79 & 13 & 11 & 337 & 43 & 8 & 15 & 9 & 14 & 16 & 19 \\
\hline A. gambiae OTOPLa & 65 & 14 & 69 & 13 & 11 & 264 & 43 & 8 & 15 & 9 & 14 & 16 & 19 \\
\hline A. mellifera. OTOPLa & 73 & 14 & 74 & 13 & 11 & 202 & 44 & 8 & 15 & 9 & 14 & 16 & 19 \\
\hline T. castaneum OTOPLa & 65 & 14 & 79 & 13 & 11 & 148 & 42 & 8 & 15 & 9 & 14 & 16 & 19 \\
\hline $\begin{array}{l}\text { D. melanogaster } \\
\text { OTOPLb }\end{array}$ & 76 & 14 & 26 & 13 & 12 & 94 & 34 & 8 & 20 & 10 & 14 & 16 & 19 \\
\hline $\begin{array}{l}\text { D. pseudoobscura } \\
\text { OTOPLb }\end{array}$ & 76 & 14 & 26 & 13 & 12 & 94 & 34 & 8 & 20 & 10 & 14 & 16 & 19 \\
\hline A. gambiae OTOPLbI & 26 & 14 & 28 & 13 & 12 & 91 & 37 & 8 & 21 & 10 & 14 & 16 & 22 \\
\hline A. aegypti OTOPLb I & 197 & 14 & 26 & 13 & 12 & 87 & 37 & 8 & 21 & 10 & 14 & 16 & 22 \\
\hline T. castaneum OTOPLb & 106 & 14 & 34 & 14 & 12 & 43 & 35 & 13 & 21 & 7 & 14 & 16 & 31 \\
\hline A. gambiae OTOPLb2 & 163 & 14 & 32 & 13 & 12 & 60 & 41 & 8 & 19 & 7 & 14 & 16 & 21 \\
\hline A. aegypti OTOPLb2 & 157 & 14 & 29 & 13 & 13 & 75 & 35 & 8 & 18 & 10 & 14 & 16 & 21 \\
\hline $\begin{array}{l}\text { D. melanogaster } \\
\text { OTOPLc }\end{array}$ & $0^{*}$ & 14 & 60 & 13 & 12 & 73 & 34 & 8 & 15 & 7 & 14 & 16 & 115 \\
\hline $\begin{array}{l}\text { D. pseudoobscura } \\
\text { OTOPLc }\end{array}$ & $0 *$ & 14 & 59 & 13 & 12 & 74 & 34 & 8 & 15 & 7 & 14 & 16 & 90 \\
\hline A. gambiae OTOPLc & $7^{*}$ & 14 & 70 & 13 & 12 & 70 & 33 & 8 & 15 & 7 & 14 & 16 & 21 \\
\hline A. aegypti OTOPLc & 10 & 14 & 68 & 13 & 12 & 70 & 33 & 8 & 15 & 7 & 14 & 16 & 21 \\
\hline A. mellifera OTOPLc & 90 & 14 & 46 & 13 & 12 & 74 & 37 & 8 & 15 & 7 & 14 & 16 & 21 \\
\hline T. castaneum OTOPLc & 177 & 14 & 50 & 13 & 12 & 79 & 33 & 8 & 15 & 7 & 14 & 16 & 21 \\
\hline C. elegans OTOPLdI & 38 & 14 & 31 & 9 & 11 & 59 & 25 & 7 & 16 & 10 & 14 & 13 & 36 \\
\hline C. briggsae OTOPLd & 47 & 14 & 34 & 9 & 11 & 59 & 25 & 7 & 16 & 10 & 14 & 13 & 37 \\
\hline C. elegans OTOPLd2 & 55 & 14 & 34 & 9 & 11 & 59 & 25 & 7 & 16 & 10 & 12 & 13 & 38 \\
\hline C. elegans OTOPLe & 50 & 14 & 36 & 9 & 11 & 69 & 22 & 7 & 16 & 11 & 14 & 13 & 34 \\
\hline C. briggsae OTOPLe & 42 & 14 & 36 & 9 & 11 & 67 & 22 & 7 & 16 & 11 & 14 & 13 & 37 \\
\hline C. elegans OTOPLf & 65 & 14 & 35 & 9 & 11 & 93 & 25 & 7 & 16 & 10 & 14 & 13 & 38 \\
\hline
\end{tabular}


Table 2: Transmembrane domain inter-loop length (amino-acids) (Continued)

\begin{tabular}{|c|c|c|c|c|c|c|c|c|c|c|c|c|c|}
\hline C. briggsae OTOPLf & 71 & 14 & 36 & 9 & 11 & 99 & 25 & 7 & 16 & 10 & 14 & 13 & 48 \\
\hline C. elegans OTOPLg & 64 & 13 & 34 & 9 & II & 72 & 26 & 7 & 16 & II & 14 & 13 & 53 \\
\hline C. briggsae OTOPLg & 64 & 13 & 34 & 9 & II & 73 & 26 & 7 & 16 & II & 14 & 13 & 55 \\
\hline C. elegans OTOPLh & 56 & 17 & 81 & 9 & II & 75 & 25 & 7 & 22 & II & 13 & 16 & 34 \\
\hline C. briggsae OTOPLh & 66 & 17 & 47 & 9 & 11 & 70 & 25 & 7 & 22 & II & 13 & 16 & 34 \\
\hline C. elegans OTOPLi & 51 & 18 & 29 & 14 & II & 46 & 27 & 5 & 22 & 10 & 16 & 16 & 30 \\
\hline C. briggsae OTOPLi & 51 & 18 & 30 & 14 & 11 & 48 & 27 & 5 & 22 & 10 & 16 & 16 & 30 \\
\hline Average & 62.4 & 13.2 & 33.7 & 10.8 & 11.2 & 70.5 & 27.6 & 8.1 & 18.2 & 10.2 & 43.1 & 15.6 & 23.2 \\
\hline SD & 37.3 & 1.5 & 17.0 & 1.6 & 0.5 & 50.2 & 6.5 & 1.2 & 2.0 & 1.4 & 30.6 & 1.1 & 18.3 \\
\hline
\end{tabular}

\#Number of amino-acid residues within the N-terminal, interloop, and C-terminal domains. (I), inner loop; (O), outer loop; SD, standard deviation.*Incomplete Nterminal sequence data were excluded from $\mathrm{N}$-terminal loop length calculations.

predicted to be cytosolic. The 12 TM domain model predicted by hydrophobicity and evolutionary constraint analysis places the proposed glycosylation sites in the extracellular space (Figure 2B), and suggests that it may reflect a more accurate version of OTOP insertion into the lipid bilayer. Interestingly, the missense mutations in the $t l t$, $m l h$, and $b k s$ animal models, which lead to functional loss of OTOP1 activity, each occur within highly conserved transmembrane domains; such mutations often alter the hydrophobicity of the conserved TM domain, which may lead to alterations in the ability of the protein to insert and orient in membranes.

Otop 1 is required for the formation of vertebrate otoconia, a process that involves calcium carbonate biomineralization and requires the regulation of intracellular calcium. Biochemical studies in transfected cells show that OTOP1 modulates the manner in which cells handle intracellular calcium in response to purinergic stimuli [11]. The mechanisms of calcium carbonate biomineralization are highly conserved in the development of otoconia and otoliths in the vertebrate inner ear, the formation of the avian egg-

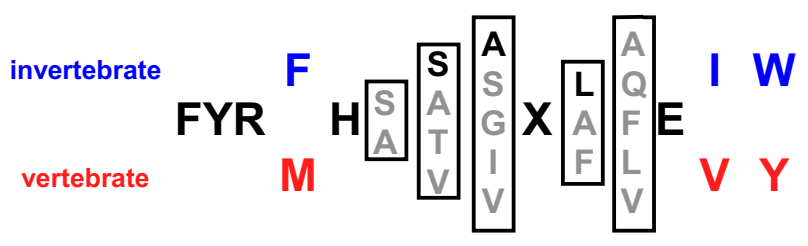

Figure 3

FYR box consensus sequence for the ODP family Cterminal tail. Residues in bold are shared by all ODP family members, $X$ is any hydrophobic amino acid, blue residues are specifically conserved in arthropod and nematode members, and red amino acids are conserved among vertebrate members. Grey, bracketed residues represent common variants at each less-conserved position. The dark residue within each bracket represents the most common amino-acid variant at that position, if one can be identified. shell, the mineralization of the arthropod exoskeleton, and the development of other mineralized structures such as the mollusk shell [21-23]. There is evidence that some ODP family members are expressed in tissues associated with calcium secretion and calcium carbonate-based mineralization. In particular, ESTs from Callinectes sapidus (Blue crab) reveal strong expression of the D. melanogaster OTOPL $b$ ortholog in hypodermal tissues that are required for calcium mobilization during the mineralization of the chitinous exoskeleton [24]. ODP mRNAs are also expressed in the hemocytes of various invertebrate species, which have been associated with the development of mineralized structures in mollusks [25]. In mammals, Otop1 is expressed in the lactating mammary gland [7], perhaps functioning in the secretion of calcium into milk. Taken together, the sequence homology, structural constraint, and expression pattern suggest a conserved role for members of the ODP family in the formation of mineralized structures. Further examination of ODPs and continued characterization of natural and induced mutations in these proteins through both physiologic and topologic studies may assist in better understanding the mechanisms of establishing and maintaining mineralized structures throughout the animal kingdom.

\section{Methods}

\section{Sequence collection}

Orthologous Otopetrin sequences were generated by a targeted sequencing approach, or identified through tBlastn searches of available whole-genome sequences. For the targeted sequencing, BAC clones were isolated from the following libraries maintained by the BACPAC Resources Center [14,26,27]: dog (Canis familiaris; RPCI-81), cow (Bos Taurus; CHORI-240), armadillo (Dasypus novemcinctus; VMRC-5), and western clawed frog (Xenopus tropicalis; CHORI-216). Specifically, each library was screened using pooled sets of oligonucleotide-based probes designed from the established sequence of the mouse Otop1 or Otop2/Otop3 subloci (on mouse Ch5B2 and Ch11E2, respectively). After isolation and mapping, a total of four BACs (accession numbers AC148430, AC149469, 
AC147459, and AC166187) were shotgun sequenced and subjected to sequence finishing, as described [28]. The complete gene structures were determined based on alignments to mouse RefSeq mRNAs or species-specific mRNA, when available. For the tBlastn searches, we used mouse Otop1, -2 , and -3 to query vertebrate genome sequences, and Drosophila OTPLa, $-b$, and $-c$ and C. elegans OTOPLd1, $-e,-f,-g,-h$, and $-i$ to query arthropod and nematode genome sequences (see Table 1 for sequence accession numbers).

\section{Alignment, phylogenic tree generation, and evolutionary constraint versus hydropathy analysis}

The initial protein sequence alignment was performed with ProbCons $[29]$, and a preliminary phylogenetic tree was built with SEMPHY [30] using only the most confidently aligned regions of the multi-sequence alignment. The sequences were then divided into smaller groups based upon their relatedness according to the tree. Each group was re-aligned with Probcons, and each of these sub-alignments was manually adjusted. ClustalW [31,32] was then used to profile-align these sub-alignments, producing the final, full alignment. The final phylogenetic tree was constructed using SEMPHY, constraining the topology to conform to SEMPHY trees built from the subalignments. 1000 bootstrap replicates were generated for each subtree as well as the final tree. The bootstrap values shown in Figure 1 are from the lowest-level tree in which the given branch occurs.

Evolutionarily constrained regions were detected essentially as described previously [12]. The final alignment and tree were used to calculate single-site evolutionary rates with the empirical Bayesian version of the program Rate4Site [33]. These single-site rate values were smoothed using sliding-windows of weighted averaging. In each 17-position-wide window, the value at the center position of the window was given the highest relative weight, and the relative weight decreased linearly for the values on either side to the edge of the window. The resulting weighted average was assigned to the position in the protein corresponding to the center of the window. To produce the evolutionary constraint profile, the rates were then converted to relative constraint by normalizing to a range between 0 and 1 , inverted by subtracting from 1 (because a region of low evolutionary rate is under high evolutionary constraint), and plotted against the position in the protein.

To produce the hydropathy profile, the hydropathy-scale value [18] for each amino acid in a column of the multisequence alignment (corresponding to a single position on the profile) was multiplied by a weighting factor that reflects the fractional contribution of the corresponding sequence to the total sequence diversity represented [17].
The hydropathy score at each position is the sum of these values. These single-position values were smoothed using the same sliding-windows weighted averaging scheme applied to the rate values above, normalized to vary between 0 and 1, and plotted against the position in the protein.

\section{Authors' contributions}

IH carried out the analysis and drafted the manuscript. JB carried out the analysis and drafted the manuscript. BH carried out the analysis and drafted the manuscript. EDG edited the manuscript. NISC Comparative Sequencing Program provided sequence data. AS edited the manuscript. DMO carried out the analysis and drafted the manuscript. All authors read and approved the final manuscript.

\section{Additional material}

\section{Additional File 1}

CLUSTALW alignment of known and predicted ODP family members. The sources of the protein sequences utilized in this alignment are listed in Table 1. Predicted TM domains are shaded (tan) and numbered TM1 to TM12. Inter-TM loops are numbered L1 to L11. Otopetrin Domains OD-I, -II, and -III are shaded in green, purple, and blue, respectively. Dashes indicate sequence gaps.

Click here for file

[http://www.biomedcentral.com/content/supplementary/14712148-8-41-S1.PDF]

\section{Acknowledgements}

This research was supported by National Institute on Deafness and Other Communication Disorders Grants DC02236 (DMO), DC06974 (IH), and in part by the Intramural Research Program of the National Human Genome Research Institute, National Institutes of Health. We thank Linda Lobos for assembling loop length data. We thank numerous people associated with the NISC Comparative Sequencing Program, in particular Robert Blakesley, Gerry Bouffard, Jennifer McDowell, Baishali Maskeri, Nancy Hansen, Morgan Park, Pamela Thomas, Alice Young and the many dedicated mapping, sequencing and finishing technicians.

\section{References}

I. Gizzi M, Ayyagari S, Khattar V: The familial incidence of benign paroxysmal positional vertigo. Acta Otolaryngol 1998, I I 8(6):774-777.

2. Oghalai JS, Manolidis S, Barth JL, Stewart MG, Jenkins HA: Unrecognized benign paroxysmal positional vertigo in elderly patients. Otolaryngol Head Neck Surg 2000, I 22(5):630-634.

3. Oas JG: Benign paroxysmal positional vertigo: a clinician's perspective. Ann N Y Acad Sci 200I, 942:20I-209.

4. Tusa RJ: Benign paroxysmal positional vertigo. Curr Neurol Neurosci Rep 200I, I(5):478-485.

5. Bronstein AM: Benign paroxysmal positional vertigo: some recent advances. Curr Opin Neurol 2003, 16(1):I-3.

6. Lane P: Tilted (tIt). Mouse News Lett 1986, 75:28.

7. Hurle B, Ignatova E, Massironi SM, Mashimo T, Rios X, Thalmann I, Thalmann R, Ornitz DM: Non-syndromic vestibular disorder with otoconial agenesis in tilted/mergulhador mice caused by mutations in otopetrin I. Hum Mol Genet 2003, I 2(7):777-789. 
8. Besson V, Nalesso V, Herpin A, Bizot JC, Messaddeq N, Romand R, Puech $A$, Blanquet $V$, Herault $Y$ : Training and aging modulate the loss-of-balance phenotype observed in a new ENU-induced allele of Otopetrin I. Biol Cell 2005, 97( 10):787-798.

9. Sollner C, Schwarz H, Geisler R, Nicolson T: Mutated otopetrin I affects the genesis of otoliths and the localization of Starmaker in zebrafish. Dev Genes Evol 2004, 2 I 4( I 2):582-590.

10. Hughes I, Blasiole B, Huss D, Warchol ME, Rath NP, Hurle B, Ignatova $\mathrm{E}$, Dickman JD, Thalmann $\mathrm{R}$, Levenson $\mathrm{R}$, et al:: Otopetrin $\mathbf{I}$ is required for otolith formation in the zebrafish Danio rerio. Dev Biol 2004, 276(2):391-402.

II. Hughes I, Saito M, Schlesinger PH, Ornitz DM: Otopetrin I activation by purinergic nucleotides regulates intracellular calcium. Proc Natl Acad Sci USA 2007, 104(29): I 2023-I 2028.

12. Simon AL, Stone EA, Sidow A: Inference of functional regions in proteins by quantification of evolutionary constraints. Proc Natl Acad Sci USA 2002, 99(5):2912-2917.

13. Thomas JW, Green ED: Comparative sequence analysis of a single-gene conserved segment in mouse and human. Mamm Genome 2003, I 4(10):673-678.

14. Thomas JW, Prasad AB, Summers TJ, Lee-Lin SQ, Maduro VV, Idol JR, Ryan JF, Thomas PJ, McDowell JC, Green ED: Parallel construction of orthologous sequence-ready clone contig maps in multiple species. Genome Res 2002, I2(8): |277- 1285 .

15. HUGO Gene Nomenclature Committee [http://
[ www.genenames.org/]

16. Przybylski D, Rost B: Alignments grow, secondary structure prediction improves. Proteins 2002, 46(2):197-205.

17. Stone EA, Sidow A: Constructing a meaningful evolutionary average at the phylogenetic center of mass. BMC Bioinformatics 2007, 8:222.

18. Kyte J, Doolittle RF: A simple method for displaying the hydropathic character of a protein. J Mol Biol 1982, I57(I): I05-132.

19. Jones DT: Improving the accuracy of transmembrane protein topology prediction using evolutionary information. Bioinformatics 2007, 23(5):538-544.

20. Persson B, Argos P: Prediction of transmembrane segments in proteins utilising multiple sequence alignments. J Mol Biol 1994, 237(2): 182-192.

21. Wilt FH: Developmental biology meets materials science: Morphogenesis of biomineralized structures. Dev Biol 2005, 280(I): I5-25.

22. Fekete DM: Developmental biology. Rocks that roll zebrafish. Science 2003, 302(5643):24I-242.

23. Hughes I, Thalmann I, Thalmann R, Ornitz DM: Mixing model systems: Using zebrafish and mouse inner ear mutants and other organ systems to unravel the mystery of otoconial development. Brain Res 2006, I 09 I(I):58-74.

24. Wheatly MG: Calcium homeostasis in crustacea: the evolving role of branchial, renal, digestive and hypodermal epithelia. J Exp Zool 1999, 283(7):620-640.

25. Mount AS, Wheeler AP, Paradkar RP, Snider D: Hemocyte-mediated shell mineralization in the eastern oyster. Science 2004, 304(5668):297-300.

26. BACPAC Resources Center [http://bacpac.chori.org]

27. Thomas JW, Touchman JW, Blakesley RW, Bouffard GG, BeckstromSternberg SM, Margulies EH, Blanchette M, Siepel AC, Thomas PJ, McDowell JC, et al.: Comparative analyses of multi-species sequences from targeted genomic regions. Nature 2003 , 424(6950): 788-793.

28. Blakesley RW, Hansen NF, Mullikin JC, Thomas PJ, McDowell JC, Maskeri B, Young AC, Benjamin B, Brooks SY, Coleman BI, et al.: An intermediate grade of finished genomic sequence suitable for comparative analyses. Genome Res 2004, I 4(I I):2235-2244.

29. Do CB, Mahabhashyam MS, Brudno M, Batzoglou S: ProbCons: Probabilistic consistency-based multiple sequence alignment. Genome Res 2005, I 5(2):330-340.

30. Friedman N, Ninio M, Pe'er I, Pupko T: A structural EM algorithm for phylogenetic inference. J Comput Biol 2002, 9(2):33 I-353.

31. Chenna R, Sugawara H, Koike T, Lopez R, Gibson TJ, Higgins DG, Thompson JD: Multiple sequence alignment with the Clustal series of programs. Nucleic Acids Res 2003, 3 I ( I 3):3497-3500.

32. Thompson JD, Higgins DG, Gibson T]: CLUSTAL W: improving the sensitivity of progressive multiple sequence alignment through sequence weighting, position-specific gap penalties and weight matrix choice. Nucleic Acids Res 1994, 22(22):4673-4680.

33. Mayrose I, Graur D, Ben-Tal N, Pupko T: Comparison of site-specific rate-inference methods for protein sequences: empirical Bayesian methods are superior. Mol Biol Evol 2004, 2I(9): $178|-| 79 \mid$

Publish with Bio Med Central and every scientist can read your work free of charge

"BioMed Central will be the most significant development for disseminating the results of biomedical research in our lifetime. "

Sir Paul Nurse, Cancer Research UK

Your research papers will be:

- available free of charge to the entire biomedical community

- peer reviewed and published immediately upon acceptance

- cited in PubMed and archived on PubMed Central

- yours - you keep the copyright 